\title{
Development of a fault diagnosis algorithm for solar panel
}

\author{
Hye-Rin Hwang ${ }^{1}$, Berm-Soo Kim ${ }^{2}$, Rui Xu ${ }^{1}$ and In-Soo Lee ${ }^{1}$ \\ ${ }^{1}$ School of Electronics Engineering, Kyungpook National University, Daegu, 41566, \\ Korea \\ ${ }^{2}$ MIJIENERTECH Co., Ltd, Daegu, Korea \\ Email:insoolee@knu.ac.kr,miji5670@naver.com
}

\begin{abstract}
In this paper, we developed a fault diagnosis algorithm of solar panel for solar street light It measures from solar panel's open voltage to load voltage at the cut-in and saves that voltages characteristic curve in memory. By comparing the steady state curve with saved curve, the solar panel's fault diagnosis can be estimated. We also designed the solar simulator for actual operating environment.
\end{abstract}

Keywords: Solar Panel; Fault Diagnosis; Solar Simulator; Characteristic Curve.

\section{Introduction}

Due to the Environmental issues, the depletion of fossil fuels, and the people against to nuclear devices recently, people focus highly on the new and renewable energy. Among them, the solar power generation make use of the semiconductor element in a solar cell which can transfer the solar energy into the electric energy. The researchers' inlands and outlands all paid highly attention on this. Solar power generation systems can be divided into grid-connected and stand-alone power system, depending on if it is connected with the electrical power generating system. In the stand-alone systems, the demand for solar street light is urgently increasing which applies to the low-power LED lamps to replace the conventional halogen security lighting [1].

A solar street light is a system using the power stored in the electric power, which charges the electric power generating system by the solar cell module in battery during the day to light the LED lamp at night. The main function of the charging control apparatus is to efficiently charge the electric power battery as well as to detect the sunrise and sunset, in order to light on and off the LED lamp. In addition, it not only protects the system from the over-charging and over-discharging which can prolong the life of the battery, but also, in order to enhance the charging efficiency, it includes the function of the PMW(Pulse Width Modulation)charging, and the brightness control of the LED lamps to 
reduce the power consumption and so on. The most important factor of the charging control system with this function is the operating stability. Except for the basic function of charging and discharging, in order to increase the utilization of the system, it is not dependent on the technology.

On one hand, the surface of solar cell module receives the incident ray to generate electricity. The power generating plant which converts the light power into electricity do not have the mechanical operation part so that it has a strong durability and has a semi-permanent life. However, the direct ray can lead to the high heat which will lower the power generation's efficiency and can also lead to the premature cracks or damage to the solar cell component. As a result, after the solar cell module is set, it will proceed simultaneously with electrical power generation. Although it is necessary to monitor the situation of electricity generating and fault, due to the variation of the sunshine amount, the climate condition, the generating state will change all the time. The fault of the solar cell module is not easy to diagnose in the field. Especially in the case of a stand-alone solar street light, compared to that is connected via an array of hundreds of power generations, it only consists of a small solar power station and one or two other arrays, it is difficult to diagnose the fault through the electricity generating capacity. And in order to calculate the amount of electricity generating, it is necessary to detect both the voltage and the current. As a result, the price will increase.

In this paper, we propose an algorithm for diagnosing whether a fault occurs in the solar cell module by monitoring the voltage of a solar cell module in the charge control apparatus applied to a stand-alone solar street lights [2]. In addition, we design the solar simulator based on a xenon lamp for actual operating environment.

\section{Algorithm Development of Fault Diagnosis of Solar Module Cells}

The solar cell module represents a non-linear V-I characteristic curve, as shown in Figure 1. Voltage in the open circuit is called an open circuit voltage (VOC), a current in the short circuit is called short circuit current (ISC). Also voltage and current generated by the maximum power (PM) refer to a maximum voltage (VM) and the maximum current (IM). Because these five elements change every time with the temperature and the sunshine amount, we apply the MPPT (Maximum Power Point Tracking) control algorithm and the like. At the same time, by measuring these elements, the current characteristic of the solar cell module can be analyzed.

In this paper, because it is not suitable for applying the MPPT algorithm, 
when we connect the open or non-short load, the voltage and the current are marked as the load voltage (VL) and the load current (IL). The fault of the solar cell module can be figured out through the power diversity of the voltage or the current when the extreme failure occurs such as a cut-line, due to the manufacturing defect.

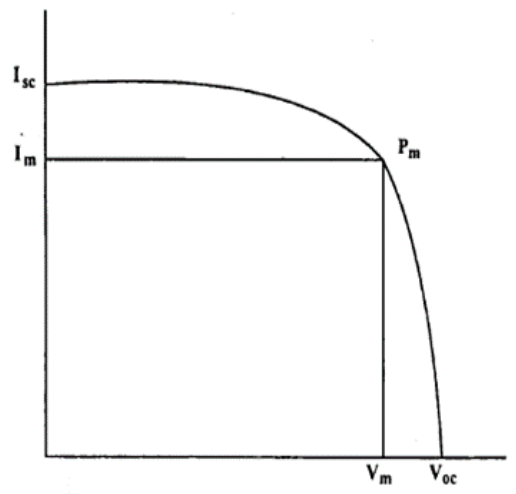

Fig. 1 Characteristic curve of the solar cell power

Fig. 2 shows the voltages and currents over time of the installed solar cell modules with the same specification at $100 \mathrm{~W}$ set in the same place. One module (M1) was placed in the open state measuring the open-circuit voltage. Another module (M2) is the same as the configuration shown in Figure 1 to measure the load voltage (VL) and the load current (IL).

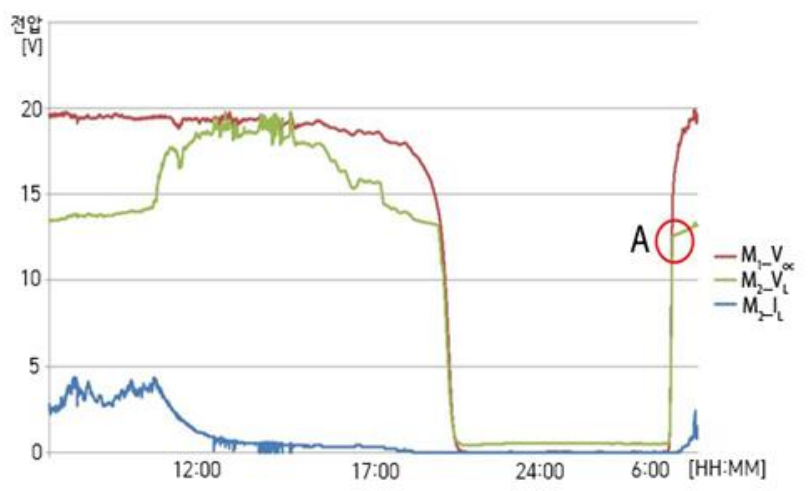

Fig. 2 Generating capacity of solar cell module

In Fig. 2, part A displays the time when the solar cell module starts to generate electricity, and can be magnified as shown in Figure 3. As observing, while 
increase the open-circuit voltage (VOC) of the modules both M1 and M2, it generates a visible point of difference. The voltage at this time is referred to as start voltage (V Cut-in). It disclosed that, when the solar cell module generates electricity, there is current flow past the load.

Therefore, when starts generating electricity, it can control the load current through the control of PWM. At the same time, Duty Ratio of PWM will gradually increase from $0 \%$ to $100 \%$ in a predetermined time interval. This means that the loads are connected from $0 \%$ to $100 \%$ in the module. In the voltage at open circuit (Voc), we can calculate the gradient of the load voltage when loads are fully connected. It is stored in the memory unit and compared to the gradient in the normal state to determine if fault occurs.

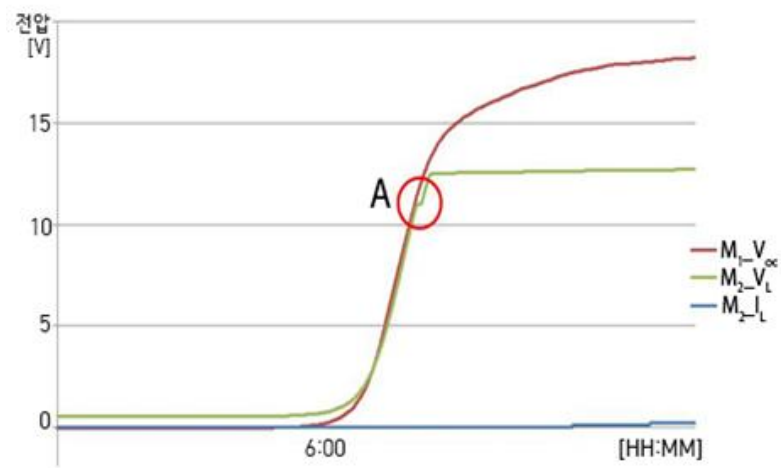

Fig. 3 The characteristic of solar power generation at start point

Referring to Figure 4, in the case of the fault due to cracks or poor electrodes cannot be judged by naked eyes. When it appears the same properties as graph $\mathrm{B}$, which compared to the graph of the steady-state $\mathrm{C}$, is significantly lower and is beyond the predetermined error rate can be estimated as a fault.

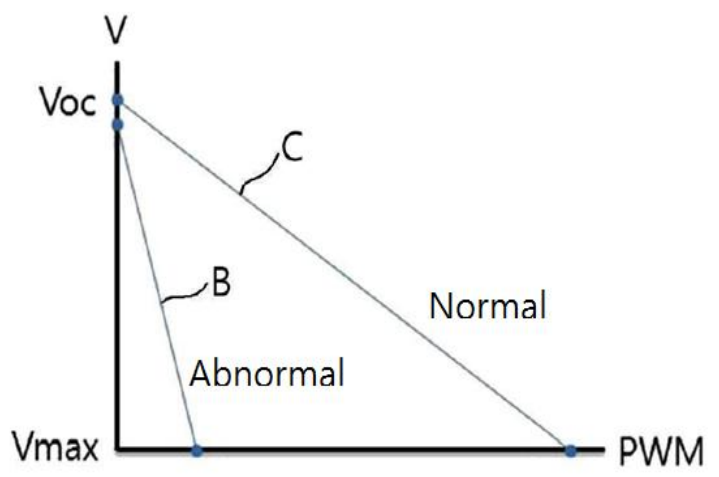

Fig. 4 Abnormal changes in the characteristic curve of module status 
The fault diagnosis section consists of the PWM controller which controls the load current, the voltage monitor which measures the load voltage and the characteristic curve generator from the open-circuit voltage to load voltage, the fault diagnosis section can diagnose the fault by comparing the error rate between the stored initial data and the immediately preceding data and the marking section which indicates the user whether or not the fault occurs.

\section{The Design of the Solar Simulator}

About the fault diagnosis algorithm mentioned above, in order to collect the actual validation data, the designer developed a solar simulator which can compare the load characteristic of the relatively normal module and the fault module. $[3,4]$ In order to value the reliability of the solar cell module in real life and in commerce, the main devices have been developed. Actually, it is not easy to collect the data of the load characteristic due to the climate changes such as the sunrise and sunset. The fault diagnosis algorithm proposed in this paper needs to analyze the contact time point of module load, so it is not applicable to the simulator used for performance valuation and software simulator.

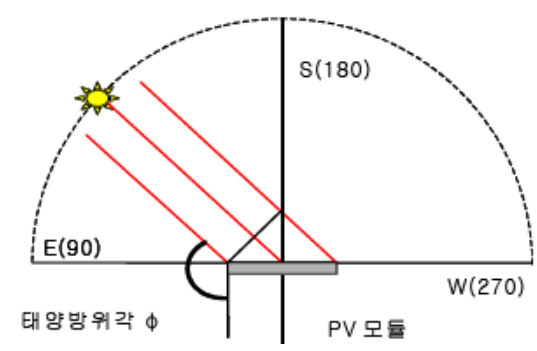

Fig. 5 The design direction of the solar simulator

As a result, using the xenon lamp as the light source which can produce precision sunlight spectrum, as shown in fig. 5. The solar module designed shape the form closest to the operating state. The light source moves from the east to the west and it can lead to the change of the module's generating capacity 


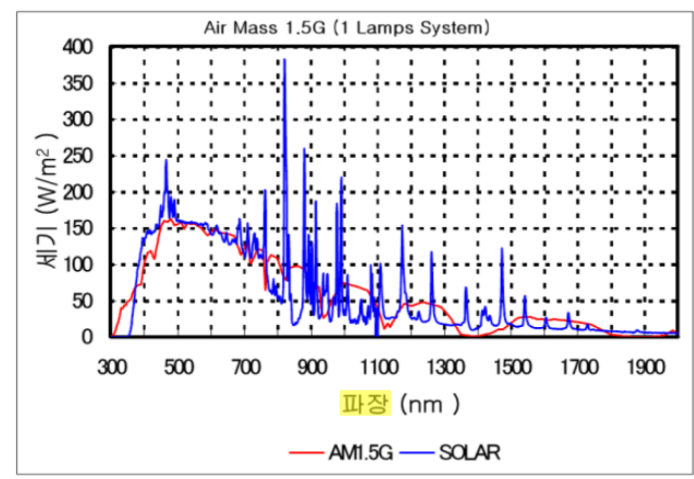

Fig. 6 Comparison of one xenon lamp and solar wavelengths

In Fig. 6, by comparing a xenon lamp and solar wavelengths, it can be seen that the error also occurs in the form of spikes above 700nm. However, considering the power consumption and the size of the entire simulation device, it is suitable for a 1000-watt xenon lamp. Considering the investigation scope of a xenon lamp, the 20-watt mono crystalline silicon solar cell module is selected. Since the temperature of the xenon lamp, the power generation capacity of the solar cell module is low. In order to avoid this situation, the height of the light and module can be selected to be $40 \mathrm{~cm}$. Light angle can be moved from the east to the west and configure the servo motor. In order to adjust to a more precise angle, it is suitable for a 1: 4 gear ratio. The design drawing is shown in Figure 7.
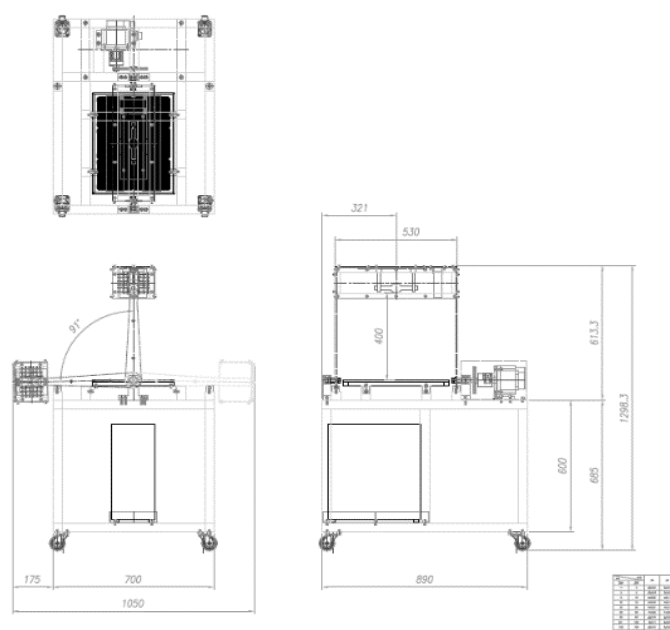

Fig. 7 Design drawing of the solar simulation device 


\section{Conclusion}

In this paper, in the solar charging control devices, the open circuit voltage and the load voltage measures the solar cell module. In addition, by storing the characteristic curve in the store unit, the characteristic curve as the base of the changing, we develop and create a simulation device which can diagnose whether the fault of the solar cell module occurs.

In addition, in an actual operating environment similar to a field and form, light will move from the east to the west. Sunrise and sunset can be observed morphology. By adjusting the xenon lamp light, a solar simulator to observe climate change has been designed. According to the position of the sun and climate change, it can collect data of normal module and fault module. Through the design of approximately 4-5 meters high sun lights, judge if fault occurs which is difficult to observe with the naked eyes. The maintainer's rapid response can improve the utilization efficiency of the system. In addition, without the current sensing unit, it is possible to achieve cost effectiveness and economical electric power calculus, so the efficiency of the controller has the advantage of gradually improvement.

\section{References}

1. C. K. Park, Study on the obsolescence forecasting judgment of PV systems adapted micro-inverters, Journal of Korea Multimedia Society, 18, 7, pp. 864-872, (2015).

2. MIJIENERTECH Co., Ltd, Apparatus and method for fault diagnosis of photovoltaic module, Patent (Korea) 10-1456122, (2014).

3. S. Y. Kang and K. H. Kim, Simulator development for stand-alone PV system design, Power Electronics Annual Conference, pp. 383-388 (Korea, 2003).

4. J. S. Lee and K. H. Kim, Solar cell engineering (Green Press, 2012). 